\title{
CLASSIFICATION OF ORCHARD CROP USING SENTINEL-1A SYNTHETIC APERTURE RADAR DATA
}

\author{
Hemant Sahu ${ }^{1, *}$, Dipanwita Haldar ${ }^{2}$, Abhishek Danodia $^{2}$, Suresh Kumar ${ }^{2}$ \\ ${ }^{1}$ Trainee, IIRS, Dehradun \& Scholar, GGV Bilaspur Chhattisgarh, India - info2hemant1980@ gmail.com \\ ${ }^{2}$ Faculty, Agriculture and Soils Department, IIRS-ISRO Dehradun India.
}

\section{Commission V, SS: Natural Resources Management}

Keywords: Sentinel-1A SAR Data, Orchard Crop, Classification algorithm, Accuracy.

\begin{abstract}
A study was conducted in Saharanpur District of Uttar Pradesh to asses the potential of Sentinel-1A SAR Data in orchard crop classification. The objective of the study was to evaluate three different classifiers that are maximum likelihood classifier, decision tree algorithm and random forest algorithm in Sentinel-1A SAR Data. An attempt is made to study Sentinel-1A SAR Data to classify orchard crop using this approach. Here the rule-based classifiers such as decision tree algorithm and random forest algorithm are compared with conventional maximum likelihood classifier. Statistical analysis of the classification show that the distribution of the crop, forest orchard, settlement and waterbody was $17.47 \%, 0.47 \%, 28.3 \%, 28.3 \%$ and $25.5 \%$ respectively in all the classification algorithm but root mean square error for maximum likelihood classifier (1.278) is more than decision tree algorithm (1.196) and random forest algorithm (1.193). Out of three, a percentage correct prediction is highest in case of decision tree algorithm (73.4) than random forest algorithm (72.5) and least for maximum likelihood classifier (66.8) in December 2017. The accuracy for orchard class is 0.81 for maximum likelihood classifier, 0.80 for decision tree algorithm and 0.78 for random forest algorithm. Thus Sentinel-1A SAR Data was effectively utilized for the classification of orchard crops.
\end{abstract}

Keywords: Sentinel-1A SAR Data, Orchard Crop, Classification algorithm, Accuracy.

\section{Introduction}

Crop discrimination is usually an important step for development and management of crop monitoring systems (Han et al., 2007). Since remote sensing technology is used for crop discrimination, both the theory and the technological tools have been in constant development, this has led to a remarkable increased in the range of applications and scope of crop discrimination techniques. The most of crop discrimination monitoring systems require as input data associated with conditions of the plants and soil, this data must not only be accurate and consistent, but must also be available in appropriate spatial and temporal scales, which is quite feasible to reach from remote platforms, such as via aircraft- and satellite-based sensor systems (Pinter et al., 2003). The capabilities of SAR for discriminating crop type have been previously explored. Studies have shown that SAR classifications were significantly improved based on a per-field approach due to the presence of speckle at the pixel level which was filtered out at the parcel level for example in rice (Haldar et al, 2012). Crop and Orchard has remained untouched for characterization and discrimination using $\mathrm{C}$ band particularly VV and VH Polarization. *

Rule based classification had certain advantages that decision tree user's ability to inspect the processes made behind the classification decisions. They are non-parametric ( $\mathrm{Lu}$ and Weng, 2007) and have been successfully demonstrated to characterize numerous targets when used in land cover classification techniques (Pal and Mather, 2003). One of the biggest advantages of random forest over decision tree is the algorithm on which the former one works i.e. it simply keeps on building tree by determining the important variable which depends on homogeneity (Prajwala, 2015). Here an attempt was made to compare the rule based advanced classifier i.e. Decision tree and Random Forest Algorithm with conventional Maximum Likelihood Classifier in Sentinel-1A SAR data The objective of the study was to evaluate three different classifiers that are maximum likelihood classifier,

\footnotetext{
* Corresponding author
}

decision tree algorithm and random forest algorithm in Sentinel1A SAR Data.

\section{Study Area}

Saharanpur is the upper most district of Uttar Pradesh separated from Dehradun district of Uttarakhand and surrounded by Shivalik hills in the north east parts. The river Yamuna forms its boundary in the west, which separates it from Karnal and Yamunanagar districts of Haryana. Saharanpur district is located in the western part of Uttar Pradesh State. It is in a rectangular shape and it lies between $29^{\circ} 34^{\prime \prime} 45^{\prime}$ and $30^{\circ}$ $21^{\prime \prime}$ North latitude and $77^{\circ}$ 9" and $78^{\circ} 14^{\prime \prime} 45^{\prime}$ East longitude (Figure 1).

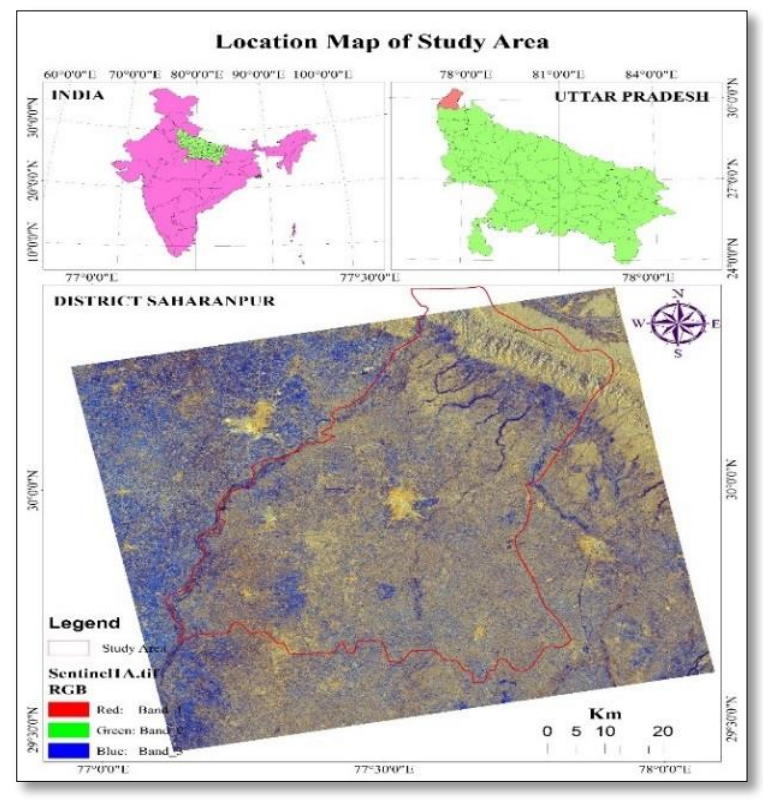

Figure 1: Location Map of Study Area

2.1 Satellite Data and Software used 
Freely available Sentinel-1A C-band IW GRD SAR data of recorded characteristics (Table 1) from the European Space Agency through Sentinels Scientific Data Hub were used for the study.

\section{Methodology}

3.1 Field Visit: Field visit was performed with scene date for ground truthing for five major feature classes namely crop forest, orchard, waterbody, and settlement. Nearly 82 sample points is covered including 34 GPS points of orchards during field visit (figure 2 and figure 3 ) as shown in table 2 .

Table 1: Main Characteristics of acquired SAR Data

\begin{tabular}{|c|c|c|}
\hline S.No & Parameter & IW \\
\hline $\mathbf{1}$ & Polarization & Dual (VV + VH) \\
\hline $\mathbf{2}$ & Access(Incidence Angle) & $31^{\circ}-46^{\circ}$ \\
\hline $\mathbf{3}$ & Azimuth resolution (m) & $<20$ \\
\hline $\mathbf{4}$ & $\begin{array}{c}\text { Ground Range resolution } \\
(\mathrm{m})\end{array}$ & $>5$ \\
\hline $\mathbf{5}$ & Swath $(\mathrm{Km})$ & $>250$ \\
\hline $\mathbf{6}$ & Maximum NESZ (dB) & -22 \\
\hline $\mathbf{7}$ & Radiometric Stability (dB) & 0.5 \\
\hline $\mathbf{8}$ & Radiometric accuracy (dB) & 1 \\
\hline $\mathbf{9}$ & Acquisition Dates & $\begin{array}{c}\text { Single Scenes } \\
(14 / 12 / 2017)\end{array}$ \\
\hline
\end{tabular}

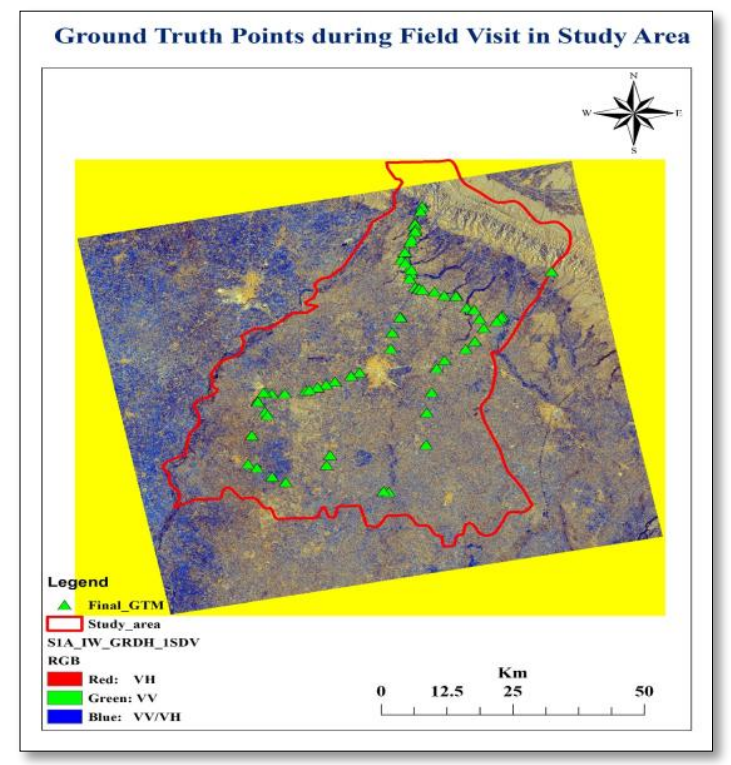

Figure 2: Ground Truthing Points

Table 2: Number of Sample Points Covered during field visit

\begin{tabular}{|c|c|c|}
\hline S.No & Feature Class & $\begin{array}{c}\text { No of Sample Points } \\
\text { Covered }\end{array}$ \\
\hline 1 & Crop & 27 \\
\hline 2 & Orchard & 4 \\
\hline 3 & Forest & 34 \\
\hline 4 & Settlement & 8 \\
\hline 5 & Waterbody & 9 \\
\hline & Total Points & 82 \\
\hline
\end{tabular}

3.2 Data Processing: Sentinel-1A IW GRD C-band multi temporal SAR dataset were freely downloaded from Copernicus Open Data hub and preprocessing has been carried out as par standard procedures including calibration, speckle filtering, terrain correction and image to image co-registration at sub pixel level (Sentinel 1 Users handbook). Generation of Region of Interest (ROI) after ground coordinate location and buffering the homogeneous pixels as features signature boundary. This ROI file was overlayed over processed SAR data as training and testing set for classification algorithm and accuracy assessment in SNAP software version 6.0.
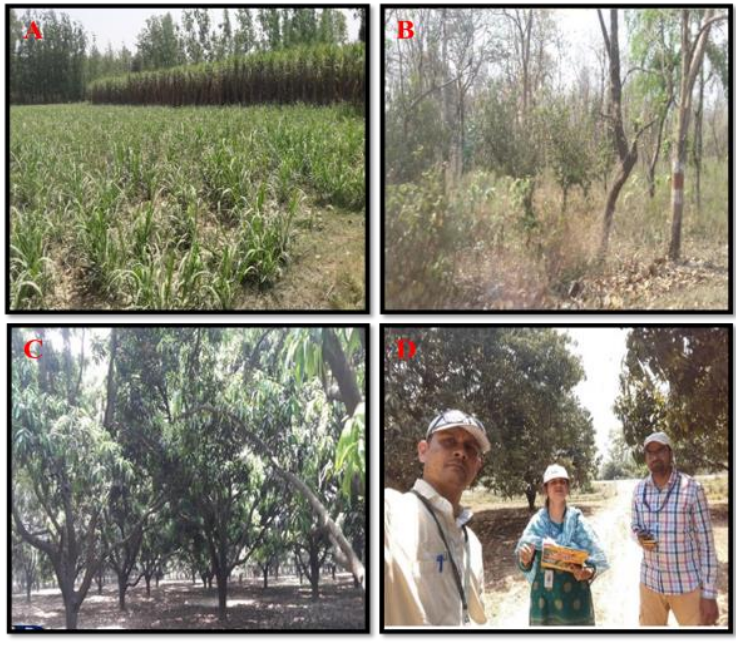

Figure 3: Sample point collection of features during field visit

A) Crop, B) Forest, C) Orchard, D) Project team

3.3 Classification Analysis: Thresholding was done to find the range and mean of backscatter coefficient. Region of Interest based classification were performed for the three selected classification techniques i.e. 1) Maximum likelihood classification (MLC), 2) Decision tree classification (KDtree$\mathrm{KNN}$ ) and 3) Random forest algorithm (RF). Rules for classification was provided in the form of vector file rather than providing manual range value for five feature class including crop, orchard, forest, settlement and waterbody.

3.4 Accuracy assessment and Statistical Analysis: Accuracy assessment was performed by evaluation of classifier using the region of interest of the features crop, orchard, forest settlement and waterbody. Results of the accuracy was subjected to comparison of accuracy, precision, correlation, error rate, rmse, bias and feature wise distributions for each of the classification algorithm. The whole methodology was summarized in the form of chart in Figure 4

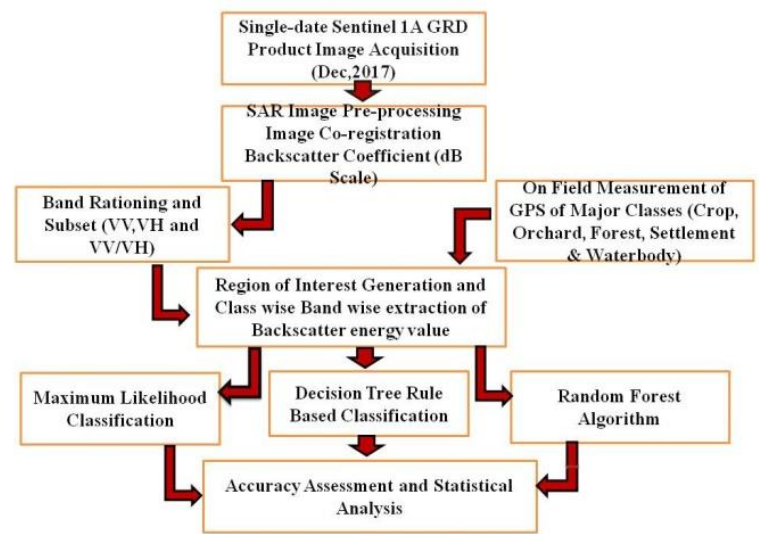

Figure 4: Flow of Methodology 


\section{Results \& Discussion}

4.1 Classification Analysis: The results of the classification were found to be different for each of the classification algorithm. The distributions of the major classes i.e. crop, forest, orchard, settlement and waterbody perform differently in each classification algorithm.

4.1.1 Maximum Likelihood Classification: In Maximum likelihood classification (MLC), the distributions of \% frequency were found to be maximum for crop (50.886\%) followed by orchard $(26.963 \%)$ and least for forest $(6.422 \%)$ as shown in figure 6A. Correlation were found to be 0.4947 for crop, 0.6336 for orchard, 0.1231 for forest , 0.6474 for settlement and 0.8958 for waterbody (Figure 5D).

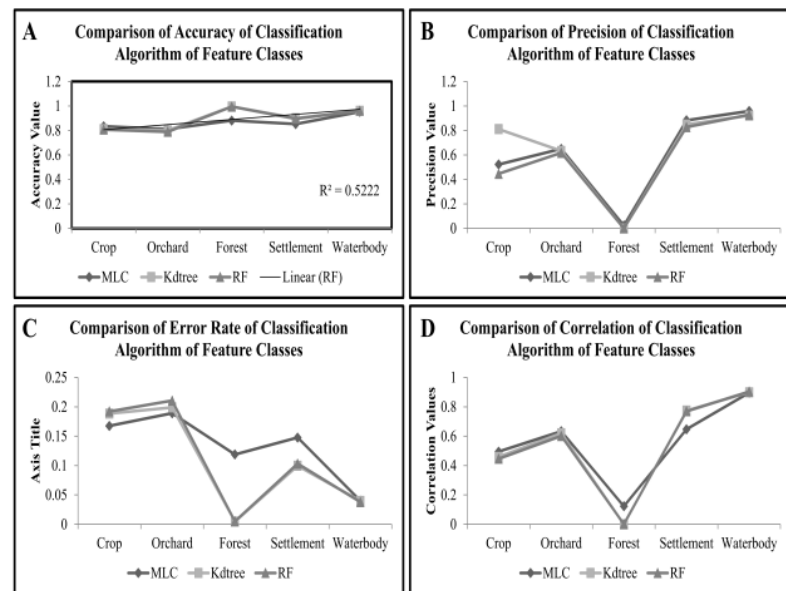

Figure 5: Comparison of the accuracy parameters MLC, KDtree and Random Forest Algorithm A) Accuracy, B) Precision, C) Error rate and D) Correlation.

4.1.2 Decision Tree Classification: In K-Decision tree algorithm (KDtree-KNN), the \% frequencies were found to be maximum for orchard $(39.715 \%)$ followed by crop $(35.015 \%)$ and least for forest $(0.019 \%)$. Correlation was found to be 0.4589 for crop, 0.62 for orchard, 0.7742 for settlement and 0.899 for waterbody.
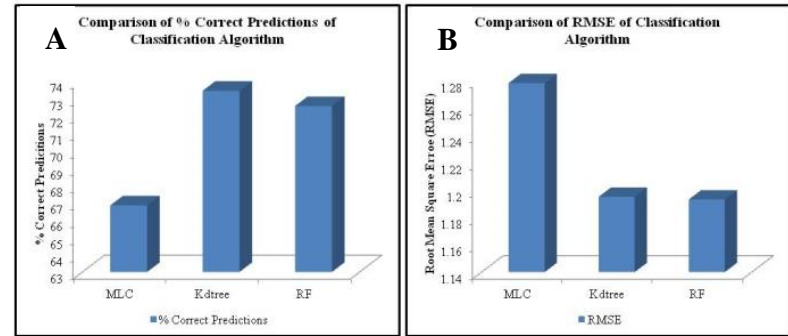

Figure 6: Comparison of Classification Algorithm A) \% Correct Predictions, B) Root mean square error (RMSE)

4.1.3 Random Forest Algorithm: In Random forest algorithm (RF), the \% frequencies were found to be maximum for orchard $(37.616 \%)$ followed by crop $(34.899 \%)$ and least for forest $(0.045 \%)$. The correlation was found to be 0.4461 for crop, 0.6013 for orchard, 0.016 for forest, 0.7696 for settlement and 0.9037 for waterbody.

4.2 Accuracy assessment and Statistical Analysis: The accuracy parameters for the entire three classification algorithm and five feature classes were compared. The accuracy was found to be maximum in MLC (0.8109) followed by KDtree (0.801) and least by RF (0.7894) for orchard as shown in Figure 5A. Precision were found to be maximum for MLC (0.6498) followed by KDtree (0.6329) and least for RF (0.617) for orchard as depicted in Figure 5B. The error rate was found to be maximum for RF (0.2106) followed by KDtree (0.199) and least for MLC (0.1891) as founded in Figure 5C. In figure 6B, among three algorithm RMSE was found to be maximum for KDtree (1.195) followed by RF (1.193). Overall the percentage correct predictions was found to maximum KDtree $(73.422 \%)$ followed by RF $(72.5446 \%)$ and least for MLC $(66.8271 \%)$ as shown in figure $6 \mathrm{~A}$. The overall distributions for each classes remains same as the distribution of the crop, forest orchard, settlement and waterbody was $17.47 \%, 0.47 \%, 28.3 \%, 28.3 \%$ and $25.5 \%$ respectively in all the three classification algorithm (Figure 7A). Accuracy for orchards was found to be maximum for MLC than KDtree and RF (Figure 7B). Classified image for three different classifier was shown in figure 8 .
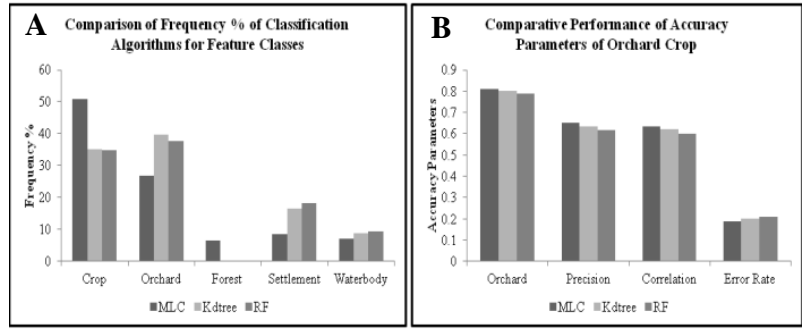

Figure 7: A) Comparison of frequency $\%$ of Classification algorithm for feature classes, B) Performance of Accuracy Parameters of Orchard Crop.

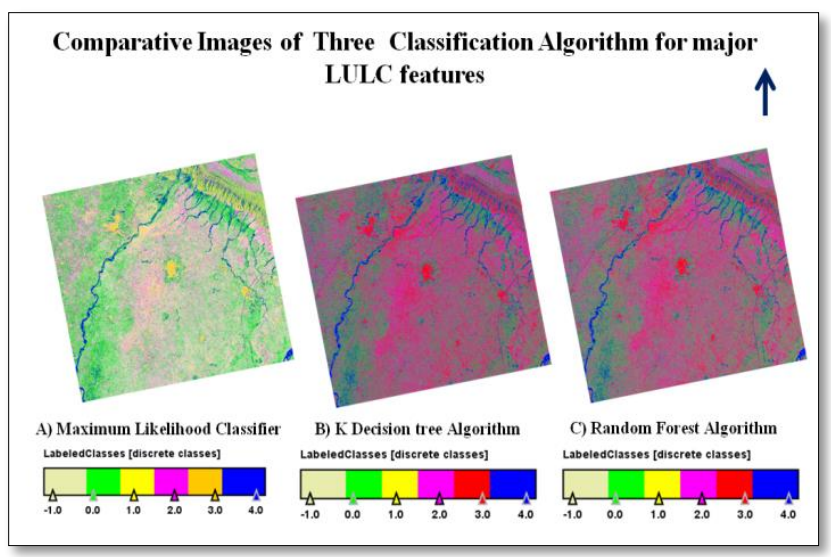

Figure 8: Classified images for three different classifiers.

5. Conclusion and Suggestions: Response of backscatter coefficient was found to be varies with different classifier used. Statistical analysis of the classification revealed that the distribution of the crop, forest orchard, settlement and waterbody was $17.47 \%, 0.47 \%, 28.3 \%, 28.3 \%$ and $25.5 \%$ respectively in all the classification algorithm but root mean square error for maximum likelihood classifier (1.278) is more than decision tree algorithm (1.196) and random forest algorithm (1.193). Out of three, a percentage correct prediction is highest in case of decision tree algorithm (73.4) than random forest algorithm (72.5) and least for maximum likelihood classifier (66.8) in December 2017. The accuracy for orchard class is 0.81 for maximum likelihood classifier, 0.80 for decision tree algorithm and 0.78 for random forest algorithm. Thus Sentinel-1A SAR Data was effectively utilized for the classification of orchard crops.

6. Acknowledgement: I would like to thank Head, Department of Rural Technology and Social Development, Guru Ghasidas Vishwavidyalaya, Bilaspur Chhattisgarh for 
providing me opportunity to work in IIRS, Dehradun and Director, IIRS-ISRO Dehradun for providing me the resources and support for carrying out this study.

\section{REFERENCES}

1. Han, L. J., Pan, Y. Z., Zhu, X. L., Wang, S., Xu, L., (2007). Acquisition of paddy rice coverage based on multi-temporal irs-p6 satellite awifs rs-data. Transactions of the CASE. 1.1.

2. Haldar, D., A. Das, S. Mohan, O. Pal, R. S. Hooda, and M. Chakraborty, (2012)."Assessment of L-band SAR data at different polarization combinations for crop and other landuse classification," Progress In Electromagnetics Research B, Vol. 36, 303-321.

3. Lu, D., \& Weng, Q. (2007). A survey of image classification methods and techniques for improving classification performance. International journal of Remote sensing, 28(5), 823-870.

4. Pal, M., \& Mather, P. M. (2003). An assessment of the effectiveness of decision tree methods for land cover classification. Remote sensing of environment, 86(4), 554565.

5. Pinter, J. P., Hatfield, L. J., Schepers, J., Barnes, E. M., Moran, S., Craig, S., Upchurch, R. D., (2003). Remote sensing for crop management. Photogrammetric Engineering and Remote Sensing. 1.1.

6. Prajwala, T. R. (2015). A comparative study on decision tree and random forest using $\mathrm{R}$ tool. International journal of advanced research in computer and communication engineering, 4(1), 196-1.

7. https://www.census2011.co.in/census/district/503saharanpur.html.

8. http://saharanpur.kvk4.in/district-profile.html

9. https://sentinel.esa.int/web/sentinel/user-guides/sentinel-1sar/product-overview/geophysical-measurements.

10. https://sentinel.esa.int/web/sentinel/user-guides/sentinel-1sar/acquisition-modes/interferometric-wide-swath.

11. https://sentinel.esa.int/web/sentinel/technical-guides/ sentinel-1-sar/products-algorithms/level-1-productformatting. 